\title{
Quantum Discord and entropic measures of quantum correlations: Optimization and behavior in finite $X Y$ spin chains
}

\author{
N. Canosa ${ }^{1}$, M. Cerezo ${ }^{1}$, N. Gigena ${ }^{1}$, R. Rossignoli ${ }^{1,2}$ \\ ${ }^{1}$ Departamento de Física-IFLP, \\ Universidad Nacional de La Plata \\ C.C. 67, La Plata (1900), Argentina \\ ${ }^{2}$ Comisión de Investigaciones Científicas (CIC), \\ La Plata (1900), Argentina
}

\begin{abstract}
We discuss a generalization of the conditional entropy and one-way information deficit in quantum systems, based on general entropic forms. The formalism allows to consider simple entropic forms for which a closed evaluation of the associated optimization problem in qudit-qubit systems is shown to become feasible, allowing to approximate that of the quantum discord. As application, we examine quantum correlations of spin pairs in the exact ground state of finite $X Y$ spin chains in a magnetic field through the quantum discord and information deficit. While these quantities show a similar behavior, their optimizing measurements exhibit significant differences, which can be understood and predicted through the previous approximations. The remarkable behavior of these quantities in the vicinity of transverse and non-transverse factorizing fields is also discussed.
\end{abstract}

\section{INTRODUCTION}

Non-classical correlations in mixed states of composite quantum systems have attracted strong attention in recent years [1, 2]. In pure states they can be identified with entanglement [3 6] and are essential for quantum teleportation [7] and for achieving exponential speed-up in pure state based quantum algorithms [8, 9]. However, in the case of mixed states it is now well known that separable states, defined in general as convex mixtures of product states 10, i.e. those which can then be created by local operations and classical communication [5, 10, may still exhibit non-classical features, such as a non-zero value of the quantum discord 11 14. The latter is defined as the difference between two distinct quantum extensions of the classical mutual information or conditional entropy, becoming zero for classically correlated states and reducing to the entanglement entropy for pure states. A finite quantum discord is also present in the mixed state based quantum algorithm of Knill and Laflamme 15], as shown in 16], which achieves an exponential speed-up over classical algorithms without substantial entanglement 17. This fact triggered the interest not only in the quantum discord and its fundamental properties 1823 but also in other related measures with similar features 1, 2], which include among others the one-way information deficit [1, 24, 26, the geometric discord 27. the generalized entropic measures introduced in 28, 29] (which contain the previous ones as particular cases), the local quantum uncertainty 30, 31], the trace distance discord 32 34 and more recently coherence based measures 2, 35, 36]. Besides, various operational interpretations of the quantum discord and other related measures have been provided 11, 19, 26, 37 41. It is worth mentioning, however, that most of these measures require the determination of an optimizing local measurement, which makes their evaluation difficult in a general situation (shown to be NP-complete 42]).
Interacting spin chains provide a useful scenario for studying the previous measures and their behavior in the vicinity of critical points 1, 43 53. In general, ground states of interacting spin chains are strongly entangled states, implying that the state of a reduced spin pair or group of spins will typically be a mixed state. Hence, for these subsystems differences between discord type measures and entanglement will arise already at zero temperature.

In this chapter we first briefly review in section II the quantum discord and the associated local measurement dependent conditional entropy on which it is based. We then discuss the consistent generalization of this entropy to general entropic forms 54, 55. This extension enables in particular the consideration of simple forms which allow an analytic solution of the associated optimization problem, i.e., that of determining the local measurement leading to the lowest conditional entropy, for general mixed states of qudit-qubit systems [54, 55. The solution is given in terms of an eigenvalue equation which admits a simple geometrical picture 54. We then examine the generalized information deficit 28], based on general entropic forms, which contains the standard one-way information deficit 24 26] as a particular case, together with its exact minimization for simple quadratic entropic forms for general states of qudit-qubit systems 27, 29].

In section III we will analyze the exact behavior of the quantum discord and the information deficit associated with spin pairs in the ground state of finite $X Y$ spin $1 / 2$ chains immersed in a magnetic field. We will show that while their behavior is quite similar, significant differences do arise in their corresponding minimizing measurements, which can be correctly predicted and understood by the approximations based on simple entropic forms. A remarkable effect in these chains is the possibility of exhibiting a completely separable exact ground state at a factorizing field. The existence of a factorizing field was first discussed in Ref. [56] and its properties 
together with the general conditions for its existence at transverse fields were analyzed in 57 66. The transverse factorizing field actually corresponds to the last ground state parity transition [61, 64, 65], and accordingly, it will be shown that in finite chains the quantum discord and information deficit exhibit full range in its vicinity, with an appreciable finite limit value at this field. We will also discuss the behavior for a non-transverse field 67, which will differ from the previous one due to the broken spin parity symmetry. Conclusions are finally given in section IV.

\section{FORMALISM}

We first describe the main features of the quantum discord and the generalized conditional entropy and information deficit, together with some analytic results for general states of qudit-qubit systems.

\section{A. Quantum Discord and conditional entropy}

Let us start with the well known quantum discord, introduced in [1], 12]. For a bipartite quantum system $A+B$ initially in a state $\rho_{A B}$, it can be defined as the minimum difference between two distinct quantum versions of the mutual information, or equivalently, of the conditional entropy:

$$
\begin{aligned}
D(A \mid B) & =\operatorname{Min}_{M_{B}}\left[I(A, B)-I\left(A, B_{M_{B}}\right)\right] \\
& =\operatorname{Min}_{M_{B}} S\left(A \mid B_{M_{B}}\right)-S(A \mid B)
\end{aligned}
$$

where the minimization is over all possible local POVM measurements [5] $M_{B}$ on $B$, characterized by a set of operators $M_{j}=I_{A} \otimes M_{j B}$ satisfying $\sum_{j} M_{j}^{\dagger} M_{j}=I_{A} \otimes I_{B}$. Here $I(A, B)=S\left(\rho_{A}\right)-S(A \mid B)$ represents the quantum mutual information 68 before the measurement and $I\left(A, B_{M_{B}}\right)=S\left(\rho_{A}\right)-S\left(A \mid B_{M_{B}}\right)$ a measurement dependent mutual information, with

$$
\begin{aligned}
S(A \mid B) & =S\left(\rho_{A B}\right)-S\left(\rho_{B}\right) \\
S\left(A \mid B_{M_{B}}\right) & =\sum_{j} p_{j} S\left(\rho_{A / j}\right)
\end{aligned}
$$

the corresponding conditional entropies, where

$$
\rho_{A / j}=p_{j}^{-1} \operatorname{Tr}_{B} \rho_{A B} M_{j}^{\dagger} M_{j}
$$

is the reduced state of $A$ after outcome $j$ at $B$, with $p_{j}=\operatorname{Tr} \rho_{A B} M_{j}^{\dagger} M_{j}$ the probability of such outcome, and $S(\rho)=-\operatorname{Tr} \rho \log _{2} \rho$ the von Neumann entropy. In the case of complete local projective measurements $M_{j}=$ $P_{j}=I_{A} \otimes P_{j B}$, with $P_{j B} \equiv\left|j_{B}\right\rangle\left\langle j_{B}\right|$ one-dimensional orthogonal projectors $\left(P_{j B} P_{k B}=\delta_{j k} P_{j B}\right)$, then

$$
S\left(A \mid B_{M_{B}}\right)=S\left(\rho_{A B}^{\prime}\right)-S\left(\rho_{B}^{\prime}\right)
$$

where $\rho_{A B}^{\prime}$ is the joint state after the (unread) local measurement,

$$
\rho_{A B}^{\prime}=\sum_{j} P_{j} \rho_{A B} P_{j}=\sum_{j} p_{j} \rho_{A / j} \otimes P_{j B}
$$

and $\rho_{B}^{\prime}=\operatorname{Tr}_{A} \rho_{A B}^{\prime}=\sum_{j} p_{j} P_{j B}$ the ensuing state of $B$.

As is well known, the mutual information $I(A, B)$ is a measure of all correlations between subsystems $A$ and $B$, being non-negative and vanishing just for product states $\rho_{A B}=\rho_{A} \otimes \rho_{B}$ [68. Eqs. (1)-(2) can then be regarded as the difference between all correlations present in the original state and the classical correlations that remain after the local measurement on $B$, measuring then the quantum correlations. Accordingly, $D(A \mid B)$ is always nonnegative 11, 12, a property which stems from the concavity of the conditional von Neumann entropy $S(A \mid B)$ 68]. It vanishes just for semi-quantum states $\rho_{A B}$, which are already of the form (7) and which then remain invariant under the local measurement determined by the projectors $P_{j B}$. The quantum discord is then non-zero not only in entangled states but also in most separable mixed states, i.e., those not of the form (7) (and hence not diagonal in a conditional product basis $\left.\left\{\left|i_{A}^{j}\right\rangle\left|j_{B}\right\rangle\right\}\right)$. For pure states $\left(\rho_{A B}^{2}=\rho_{A B}\right)$ it reduces to the entanglement entropy $S\left(\rho_{A}\right)=S\left(\rho_{B}\right)$ of the system, as $S\left(A \mid B_{M_{B}}\right)=0$ for any measurement based on rank one projectors.

We remark that in the general case, the minimum in Eq. (2) is always reached for measurements based on rank one projectors $M_{j B} \propto P_{j B}$, not necessarily orthogonal 1, 54, 55], with a minimization based on standard projective measurements, Eq. (6), providing normally a good approximation. Nevertheless, the minimization in Eq. (2) is in general still difficult, being in fact an NPcomplete problem 42.

\section{B. Generalized conditional entropy after a local measurement}

Due the previous difficulty, and in order to obtain a more clear picture of the optimization problem associated with the quantum discord, it is convenient to consider more simple entropic forms, which may enable an easier evaluation of the minimum conditional entropy. We then consider first the generalized conditional entropy [54, 5.5.

$$
S_{f}\left(A \mid B_{M_{B}}\right)=\sum_{j} p_{j} S_{f}\left(\rho_{A / j}\right)
$$

where $S_{f}(\rho)=\operatorname{Tr} f(\rho)$ is a generalized trace form entropy 68, 69]. Here $f:[0,1] \rightarrow \mathbb{R}$ is a smooth strictly concave function satisfying $f(0)=f(1)=0$, such that $S_{f}(\rho) \geq$ 0 , with $S_{f}(\rho)=0$ just for pure states. Concavity of $f$ implies, for $S_{f}(A) \equiv S_{f}\left(\rho_{A}\right)$ [54],

$$
S_{f}(A) \geq S_{f}\left(A \mid B_{M_{B}}\right)
$$

so that the average conditional mixedness of $A$ after measurement is never greater than the original mixedness, 
irrespective of the measure $S_{f}$ used to quantify it. Moreover, the minimum of $S_{f}\left(A \mid M_{B}\right)$ is also always reached for rank one projectors $M_{j B} \propto P_{j B}$ [55], as in the von Neumann case. Hence, these properties remain valid for general concave functions $f$.

In particular, we may consider simple entropic forms, like the quadratic entropy

$$
S_{2}(\rho)=2\left[1-\operatorname{Tr} \rho^{2}\right]
$$

which follows from $f_{2}(\rho)=2 \rho(1-\rho)$ and is also known as linear entropy since it corresponds to the linear approximation $-\rho \ln \rho \approx \rho(1-\rho)$. It is a particular case of the Tsallis entropies [70] $S_{q}(\rho)=\frac{1-\operatorname{Tr} \rho^{q}}{1-2^{1-q}}$, obtained for $f_{q}(\rho) \propto \rho-\rho^{q}, q>0$, which approach the von Neumann entropy for $q \rightarrow 1$ (we set $S_{f}(\rho)=1$ for a maximally mixed single qubit state).

Eq. (9) is just a linear function of the purity $\operatorname{Tr} \rho^{2}$ and does not require the explicit knowledge of the eigenvalues of $\rho$, thus enabling an easier evaluation, both theoretically and experimentally 71 73. For instance, writing a general mixed state of a system with Hilbert space dimension $d$ as

$$
\rho=\frac{1}{d}(I+\boldsymbol{r} \cdot \boldsymbol{\sigma})
$$

where $\boldsymbol{\sigma}=\left(\sigma_{1}, \ldots, \sigma_{d^{2}-1}\right)$ is an orthogonal basis for traceless operators in the system $\left(\operatorname{Tr} \sigma_{\mu}=0, \operatorname{Tr} \sigma_{\mu} \sigma_{\nu}=\right.$ $\left.d \delta_{\mu \nu}\right)$, implying $\boldsymbol{r}=\operatorname{Tr} \rho \boldsymbol{\sigma}=\langle\boldsymbol{\sigma}\rangle$, we obtain the explicit expression

$$
S_{2}(\rho)=\frac{2}{d}\left(d-1-|\boldsymbol{r}|^{2}\right)
$$

Eq. (11) shows that $|\boldsymbol{r}|^{2} \leq d-1$, with $|\boldsymbol{r}|^{2}=d-1$ just for pure states.

\section{The qudit-qubit case}

Let us now consider a composite system where $A$ is a system with Hilbert space dimension $d_{A}$ and $B$ a single qubit. Denoting with $\boldsymbol{\sigma}_{A}$ an orthogonal basis for operators in $A$ and $\boldsymbol{\sigma}_{B} \equiv \boldsymbol{\sigma}$ the Pauli matrices of $B$, a general state of this system can be written as 54.

$$
\rho_{A B}=\rho_{A} \otimes \rho_{B}+\frac{1}{2 d_{A}} \sum_{\mu, \nu} C_{\mu \nu} \sigma_{A \mu} \otimes \sigma_{B \nu}
$$

where $\rho_{A}=\frac{1}{d_{A}}\left(I_{A}+\boldsymbol{r}_{A} \cdot \boldsymbol{\sigma}_{A}\right), \rho_{B}=\frac{1}{2}\left(I_{2}+\boldsymbol{r}_{B} \cdot \boldsymbol{\sigma}\right)$ are the reduced states of $A$ and $B$, with $\boldsymbol{r}_{A}=\left\langle\boldsymbol{\sigma}_{A}\right\rangle, \boldsymbol{r}_{B}=\langle\boldsymbol{\sigma}\rangle$, and

$$
C_{\mu \nu}=\left\langle\sigma_{A \mu} \otimes \sigma_{B \nu}\right\rangle-\left\langle\sigma_{A \mu}\right\rangle\left\langle\sigma_{B \nu}\right\rangle
$$

are the elements of the correlation tensor, represented by the $\left(d_{A}^{2}-1\right) \times 3$ matrix $C$.

We consider a local POVM measurement on the qubit $B$ based on rank one operators $M_{\boldsymbol{k} B}=\sqrt{q_{\boldsymbol{k}}} P_{\boldsymbol{k} B}$, where
$P_{\boldsymbol{k} B}=\frac{1}{2}\left(I_{2}+\boldsymbol{k} \cdot \boldsymbol{\sigma}\right)$, with $\boldsymbol{k}$ a unit vector $(|\boldsymbol{k}|=1)$, is the projector onto the pure qubit state with $\langle\boldsymbol{\sigma}\rangle=\boldsymbol{k}$, and $\sum_{\boldsymbol{k}} q_{\boldsymbol{k}} P_{\boldsymbol{k} B}=I_{2}$. We may then express the ensuing conditional entropy (8) as

$$
S_{f}\left(A \mid B_{\boldsymbol{k}}\right)=\sum_{\boldsymbol{k}} p_{\boldsymbol{k}} S_{f}\left(\rho_{A / \boldsymbol{k}}\right)
$$

where

$$
\begin{aligned}
\rho_{A / \boldsymbol{k}} & =\rho_{A}+\frac{1}{d_{A}}\left(\frac{C \boldsymbol{k}}{1+\boldsymbol{r}_{B} \boldsymbol{k}}\right) \cdot \boldsymbol{\sigma}_{A} \\
p_{\boldsymbol{k}} & =\frac{1}{2} q_{\boldsymbol{k}}\left(1+\boldsymbol{r}_{B} \cdot \boldsymbol{k}\right)
\end{aligned}
$$

are, respectively, the conditional post measurement state of $A$ after result $\boldsymbol{k}$ and the probability of obtaining this result. The vector $\boldsymbol{r}_{A / \boldsymbol{k}}$ characterizing the postmeasurement state of $A$ is then

$$
\boldsymbol{r}_{A / \boldsymbol{k}}=\boldsymbol{r}_{A}+\frac{C \boldsymbol{k}}{1+\boldsymbol{r}_{B} \cdot \boldsymbol{k}} .
$$

For a standard projective spin measurement along direction $\boldsymbol{k}$ just vectors $\pm \boldsymbol{k}$ are to be considered in the previous sums, with $q_{ \pm \boldsymbol{k}}=1$.

While in the general case the eigenvalues of $\rho_{A / \boldsymbol{k}}$ are required for the evaluation of (14), for the quadratic entropy (9) a closed evaluation is directly feasible with Eq. (11). For a standard projective spin measurement along direction $\boldsymbol{k}$ we obtain 54

$$
\begin{aligned}
S_{2}\left(A \mid B_{\boldsymbol{k}}\right) & =S_{2}\left(\rho_{A}\right)-\Delta S_{2}\left(A \mid B_{\boldsymbol{k}}\right), \\
\Delta S_{2}\left(A \mid B_{\boldsymbol{k}}\right) & =\frac{2}{d_{A}} \frac{|C \boldsymbol{k}|^{2}}{1-\left(\boldsymbol{r}_{B} \cdot \boldsymbol{k}\right)^{2}}=\frac{2}{d_{A}} \frac{\boldsymbol{k}^{T} C^{T} C \boldsymbol{k}}{\boldsymbol{k}^{T} N_{B} \boldsymbol{k}}
\end{aligned}
$$

where $C^{T} C$ and $N_{B}=I-\boldsymbol{r}_{B} \boldsymbol{r}_{B}^{T}$, are $3 \times 3$ positive semi-definite matrices. Eq. (19) is non-negative and independent of $\boldsymbol{r}_{A}$, and represents the average conditional purity gain due to the measurement on $B$. Since Eq. (19) is a ratio of quadratic forms, the direction $\boldsymbol{k}$ which leads to the maximum entropy decrease, i.e. to the minimum conditional entropy, can be obtained by solving the generalized eigenvalue equation 54]

$$
C^{T} C \boldsymbol{k}=\lambda N_{B} \boldsymbol{k},
$$

which implies $\operatorname{Det}\left[C^{T} C-\lambda N_{B}\right]=0$, and selecting the eigenvector $\boldsymbol{k}$ associated with the largest eigenvalue $\lambda_{\max }$. This leads to $\Delta S_{2}\left(A \mid B_{\boldsymbol{k}}\right) \leq 2 \lambda_{\max } / d_{A} \forall \boldsymbol{k}$, i.e.,

$$
\operatorname{Min}_{\boldsymbol{k}} S_{2}\left(A \mid B_{\boldsymbol{k}}\right)=S_{2}\left(\rho_{A}\right)-\frac{2}{d_{A}} \lambda_{\max } .
$$

An important remark is that generalized POVM measurements on qubit $B$ cannot decrease the projective minimum (21) for this entropy 54.

It is then seen that the minimizing measurement is essentially determined by the correlation tensor (13), i.e., it is essentially a spin measurement along the direction of maximum correlation. We may also express (19) as 


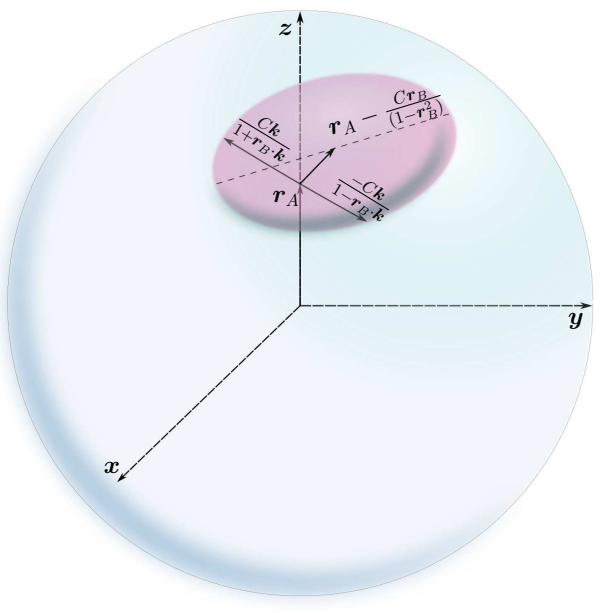

FIG. 1. The set of possible Bloch vectors $\boldsymbol{r}_{A / \boldsymbol{k}}$ of the postmeasurement state of qubit $A$ after a measurement on the qubit $B$ form the correlation ellipsoid (from [54]). For a spin measurement along direction $\boldsymbol{k}$ at qubit $B$, the vectors $\boldsymbol{r}_{A / \pm \boldsymbol{k}}$ in $A$ are the endpoints of a chord running through $\boldsymbol{r}_{A}$. The optimizing measurement determined by Eq. (20) leads to $\delta \boldsymbol{r}_{A}$ parallel to the major axis of this ellipsoid.

the quadratic form $\Delta S_{2}\left(A \mid B_{\boldsymbol{k}}\right)=\frac{2}{d_{A}} \boldsymbol{k}_{N}^{T} C_{N}^{T} C_{N} \boldsymbol{k}_{N}$, where $C_{N}=C N_{B}^{-1 / 2}$ and $\boldsymbol{k}_{N}=N_{B}^{1 / 2} \boldsymbol{k} /\left|N_{B}^{1 / 2} \boldsymbol{k}\right|$, and write (20) as $C_{N}^{T} C_{N} \boldsymbol{k}_{N}=\lambda \boldsymbol{k}_{N}$, which shows that $\sqrt{\lambda_{\max }}$ is the maximum singular value of $C_{N}$. The counterpart at $A$ of this equation is $C_{N} C_{N}^{T} \boldsymbol{k}_{A}=\lambda \boldsymbol{k}_{A}$, which has the same non-zero eigenvalues and provides a clear geometric picture: As $\boldsymbol{k}$ is varied in the Bloch sphere of qubit $B$, the set of post-measurement vectors (17) determining the post-measurement state of $A$ form a three dimensional correlation ellipsoid on the $d_{A}^{2}-1$ dimensional space containing the vector $\boldsymbol{r}_{A / \boldsymbol{k}}$ [54] (see Fig. 1 1 for the two-qubit case) whose principal axes are precisely determined as the eigenvectors $\boldsymbol{k}_{A}$ of the previous equation. Therefore, the optimizing measurement of the quadratic entropy is that leading to $\delta \boldsymbol{r}_{A}=\boldsymbol{r}_{A}-\boldsymbol{r}_{A / \boldsymbol{k}} \propto C \boldsymbol{k}$ parallel to the major semi-axis of the correlation ellipsoid (see 54 for more details).

While not strictly valid for other entropies, these results provide an approximate picture of the measurement minimizing the conditional entropy in these systems, which will typically lie close to that minimizing the quadratic entropy. In fact, all entropies $S_{f}(\rho)$ reduce essentially to the quadratic entropy if $\rho$ is sufficiently close to maximum mixedness, as $S_{f}\left(\frac{I}{d}+\delta \rho\right) \approx$ $S_{f}\left(\frac{I_{d}}{d}\right)+\frac{1}{4}\left|f^{\prime \prime}\left(\frac{1}{d}\right)\right|\left[\left(S_{2}\left(\frac{I}{d}+\delta \rho\right)-S_{2}\left(\frac{I_{d}}{d}\right)\right]\right.$ up to $O\left(\delta \rho^{2}\right)$. Moreover, for a sufficiently small correlation tensor, i.e. if $\left|\delta \boldsymbol{r}_{A}\right|=\left|\frac{C \boldsymbol{k}}{1 \pm \boldsymbol{r}_{B} k}\right| \ll 1 \forall \boldsymbol{k}$, an expansion of the conditional entropy (8) up to second order in $\delta \rho_{A}$ leads to 54 .

$$
S_{f}\left(A \mid B_{\boldsymbol{k}}\right) \approx S_{f}\left(\rho_{A}\right)-\frac{2}{d_{A}} \frac{\boldsymbol{k}^{T} C^{T} \Lambda_{f}\left(\rho_{A}\right) C \boldsymbol{k}}{\boldsymbol{k}^{T} N_{B} \boldsymbol{k}}
$$

where $\Lambda_{f}\left(\rho_{A}\right)$ is a scaled $\left(d_{A}^{2}-1\right) \times\left(d_{A}^{2}-1\right)$ Hessian ma- trix 54, showing that in the present weakly correlated regime the effect of a general entropy is just to replace $C$ by the "deformed" correlation tensor $C_{f}=\sqrt{\Lambda_{f}\left(\rho_{A}\right)} C$. Let us finally mention that the minimum generalized conditional entropy coincides with the associated generalized entanglement of formation between $A$ and a third system $C$ purifying the whole system [18, 55].

\section{Generalized information deficit}

As mentioned in the introduction, several other measures of quantum correlations with properties similar to those of the quantum discord have been considered. In particular, we have introduced in 28, 29] the generalized information deficit

$$
I_{f}^{B}\left(\rho_{A B}\right)=\operatorname{Min}_{M_{B}} S_{f}\left(\rho_{A B}^{\prime}\right)-S_{f}\left(\rho_{A B}\right),
$$

where $\rho_{A B}^{\prime}$ is the state of the system after an unread local measurement at $B$, Eq. (7), and the minimization is over all complete local projective measurements on B. Here $S_{f}(\rho)$ denotes a generalized entropy. In the case of the von Neumann entropy $S(\rho)$, Eq. 23) becomes the standard one-way information deficit [1, 24, 26], which will be denoted as $I_{1}^{B}$. It can be rewritten in terms of the relative entropy [68, 74] $S\left(\rho \| \rho^{\prime}\right)=-\operatorname{Tr} \rho\left(\log _{2} \rho^{\prime}-\log _{2} \rho\right)$ as

$$
I_{1}^{B}\left(\rho_{A B}\right)=\operatorname{Min}_{M_{B}} S\left(\rho_{A B}^{\prime}\right)-S\left(\rho_{A B}\right)=\operatorname{Min}_{M_{B}} S\left(\rho_{A B} \| \rho_{A B}^{\prime}\right) .
$$

Like the quantum discord, Eq. 23) (and hence (24)) is non-negative if $S_{f}(\rho)$ is Schur-concave 75], due to the majorization relation 28, 68] $\rho_{A B}^{\prime} \prec \rho_{A B}$ satisfied by the post-measurement state (77). Essentially, the offdiagonal elements of $\rho_{A B}$ in the conditional product basis $\left\{\left|i_{A}^{j}\right\rangle\left|j_{B}\right\rangle\right\}$ where $\rho_{A B}^{\prime}$ is diagonal are lost in the measurement, and Eq. (23) is then a measure of the minimum information loss under such measurement. It can also be considered as the minimum relative entropy of coherence 35] in this type of basis. And it is a measure of the minimum entanglement between the measurement device and the system generated by a complete local measurement 76], with (24) representing the minimum distillable entanglement 26, 39].

For strict concavity of $S_{f}$, Eq. (23) vanishes only if $\rho_{A B}$ is already of the semi-quantum post-measurement form (7). And for pure states $\rho_{A B}=\left|\Psi_{A B}\right\rangle\left\langle\Psi_{A B}\right|$ it can be shown 28] that it reduces to the corresponding entanglement entropy:

$$
I_{f}^{B}\left(\left|\Psi_{A B}\right\rangle\right)=S_{f}\left(\rho_{A}\right)=S_{f}\left(\rho_{B}\right)
$$

with (24) becoming the standard entanglement entropy like the quantum discord. Nonetheless, unlike the latter (which in this case is minimized by a complete measurement in any local basis) the minimum of (23) and (24) for a pure state is always reached for a measurement in the 
basis of $B$ which corresponds to the Schmidt decomposition of $\left|\Psi_{A B}\right\rangle$ (and hence diagonalizes $\rho_{B}$ ) 28], already indicating a different behavior of the minimizing measurement.

As in the case of the conditional entropy, the use of generalized entropies enables the possibility of using simple entropic forms like the quadratic entropy (9) or the Tsallis entropies, in which case Eq. (23) becomes $I_{q}^{B}\left(\rho_{A B}\right)=\operatorname{Min}_{M_{B}} \frac{\operatorname{Tr}\left(\rho_{A B}^{q}-\rho_{A B}^{q}\right)}{1-2^{1-q}}$. We may also consider the deficits based on the Renyi entropies 68 $S_{R_{q}}(\rho)=\frac{1}{1-q} \log _{2} \operatorname{Tr} \rho^{q}, q>0$ (just an increasing function of $\left.S_{q}\right)$, which are given by [76]

$$
I_{R_{q}}^{B}\left(\rho_{A B}\right)=\operatorname{Min}_{M_{B}} \frac{1}{1-q} \log _{2} \frac{\operatorname{Tr}{\rho^{\prime}}_{A B}^{q}}{\operatorname{Tr} \rho_{A B}^{q}} .
$$

They approach the von Neumann information deficit (24) for $q \rightarrow 1$ and likewise do not depend on the addition of an uncorrelated ancilla to $A\left(\rho_{A B} \rightarrow \rho_{C} \otimes \rho_{A B}\right)$. Nonetheless, they are just increasing functions of $I_{q}^{B}$ for fixed $\rho_{A B}$ and the associated optimization problem is the same as that for $I_{q}^{B}$.

\section{E. Minimizing measurement and stationary conditions}

The determination of the minimizing measurement $M_{B}$ in (23) is, like in the case of the quantum discord, again a difficult problem in general. Complete projective measurements at $B$ are determined by $d_{B}^{2}-d_{B}$ real parameters if $B$ has Hilbert space dimension $d_{B}$, growing then exponentially with the number of components of $B$. Nevertheless, it can be shown that the minimizing measurement should fulfill the stationary condition 29]

$$
\operatorname{Tr}_{A}\left[f^{\prime}\left(\rho_{A B}^{\prime}\right), \rho_{A B}\right]=0,
$$

which leads to $d_{B}\left(d_{B}-1\right)$ real equations 29, 77]. In the quantum discord (10), an additional term $-\left[f^{\prime}\left(\rho_{B}^{\prime}\right), \rho_{B}\right]=$ $\left[\log _{2} \rho_{B}^{\prime}, \rho_{B}\right]$ is to be added in (27) for complete projective measurements 29].

Important differences between the measurements minimizing $I_{f}^{B}\left(\rho_{A B}\right)$ and $D(A \mid B)$ may arise, as previously mentioned for the case of pure states. While for a general classically correlated state of the form (7) the minimum for both $D(A \mid B)$ and all $I_{f}^{B}\left(\rho_{A B}\right)$ is attained for a measurement in the local basis defined by the projectors $P_{j}^{B}$ (i.e., the pointer basis 11, 12]), in the particular case of product states $\rho_{A} \otimes \rho_{B}, D(A \mid B)$ (but not $\left.I_{f}^{B}\left(\rho_{A B}\right)\right)$ becomes the same for any $M_{B}$, as for such states $S\left(A \mid M_{B}\right)=S(A) \forall M_{B}$. These differences will have important consequences in the results of the next section, leading to a quite different response of the minimizing measurement to the onset of quantum correlations. They reflect the fact that while in $I_{f}^{B}\left(\rho_{A B}\right)$ one is looking for the least disturbing local measurement, such that $\rho_{A B}^{\prime}$ is as close as possible to $\rho_{A B}$, in $D(A \mid B)$ the search is for the measurement in $B$ which makes the ensuing conditional entropy smallest, i.e., by which one can learn the most about $A$, which leads to those observables which are most correlated, as discussed before.

These differences become apparent in the case of the quadratic entropy (9), as an analytic evaluation of the associated deficit $I_{2}^{B}$ for qudit-qubit systems becomes again feasible [27, 29]. In this case Eq. (23) becomes just a purity difference, $I_{2}^{B}\left(\rho_{A B}\right)=2 \operatorname{Min}_{M_{B}} \operatorname{Tr}\left(\rho_{A B}^{2}-\rho_{A B}^{\prime 2}\right)=$ $2 \operatorname{Min}_{\rho_{A B}^{\prime}}\left\|\rho_{A B}-\rho_{A B}^{\prime}\right\|^{2}$, where $\|O\|^{2}=\operatorname{Tr} O^{\dagger} O$ and the last minimization can be extended to any state of the general form (77). Through the last expression it is seen that it is then proportional to the geometric discord [1, 27, defined as the closest squared Hilbert-Schmidt distance between $\rho_{A B}$ and a state of the form (7). For pure states $I_{2}^{B}$ becomes the squared concurrence $C_{A B}^{2}$ 78], which for such states is just the quadratic entropy of any of the subsystems [79]. While as a measure it does not comply, due to the lack of additivity, with all the properties satisfied by the quantum discord or the von Neumann based information deficit, it has the advantage of enabling a simple analytic evaluation in qudit-qubit systems and admitting through its relation with the purity a more direct experimental access 71 73. Moreover, the optimizing measurement will be the same measurement as that minimizing the associated Renyi deficit $I_{R_{2}}^{B}\left(\rho_{A B}\right)$.

Writing again a general $\rho_{A B}$ of a qudit-qubit system in the form (12), it can be shown that for a projective spin measurement at $B$ along direction $\boldsymbol{k}$, the quadratic information loss becomes 27, 29]

$$
I_{2}^{B}(\boldsymbol{k})=\frac{1}{d_{A}}\left(\left\|\boldsymbol{r}_{B}\right\|^{2}+\|J\|^{2}-\boldsymbol{k}^{T} M_{2} \boldsymbol{k}\right)
$$

where $M_{2}$ is the positive semi-definite matrix

$$
M_{2}=\boldsymbol{r}_{B} \boldsymbol{r}_{B}^{T}+J^{T} J
$$

with $J=C+\boldsymbol{r}_{A} \boldsymbol{r}_{B}^{T}$, i.e., $J_{\mu \nu}=\left\langle\sigma_{A \mu} \otimes \sigma_{\nu}\right\rangle$. Minimization of $I_{2}^{B}(\boldsymbol{k})$ leads then to the standard eigenvalue equation $M_{2} \boldsymbol{k}=\lambda \boldsymbol{k}$, implying $I_{2}^{B}\left(\rho_{A B}\right)=\frac{1}{d_{A}}\left(\operatorname{tr} M_{2}-\lambda_{\max }\right)$, with $\lambda_{\max }$ the largest eigenvalue of $M_{2}$ and the minimizing $\boldsymbol{k}$ the associated eigenvector. Such direction will not necessarily coincide with that minimizing the quadratic conditional entropy, as the latter is determined essentially by the correlation tensor $C$ while the present one by the tensor $J$ and $\boldsymbol{r}_{B}$. While coinciding in some regimes (they become identical if $\boldsymbol{r}_{B}=\mathbf{0}$, i.e., $\rho_{B}$ maximally mixed, in which case $J=C$ ), they can deviate considerably in others, as will be explicitly shown in the next section. In fact, a transition in the least disturbing measurement direction $\boldsymbol{k}$ from the main eigenvector of $J^{T} J$ to the direction of $\boldsymbol{r}_{B}$ can be expected as $J$ decreases, which may not imply a concomitant change in the main eigenvector of (20). A closed expression for the minimum of $I_{3}^{B}\left(\rho_{A B}\right)$ can also be obtained [29]. We finally note that for a general qubit-qubit state and entropy $S_{f}$, the stationary condition (27) becomes explicitly

$$
\left(\alpha_{1} \boldsymbol{r}_{B}+\alpha_{2} J^{T} \boldsymbol{r}_{A}+\alpha_{3} J^{T} J\right) \boldsymbol{k}=\lambda \boldsymbol{k}
$$


which represents a non-linear eigenvalue equation since the coefficients $\alpha_{i}$ depend on $f^{\prime}\left(\rho_{A B}^{\prime}\right)$ and hence on $\boldsymbol{k}$ 29]. Again, the prominent role of $J^{T} J$ is clearly evident.

\section{RESULTS IN SPIN CHAINS}

We now consider the correlations of spin pairs in the ground state (GS) of finite spin 1/2 arrays interacting through $X Y$ type Heisenberg couplings and immersed in a magnetic field $\boldsymbol{h}$. The Hamiltonian reads

$$
H=-\sum_{i} \boldsymbol{h} \cdot \boldsymbol{S}_{i}-\frac{1}{2} \sum_{i \neq j, \mu=x, y} J_{\mu}^{i j} S_{i}^{\mu} S_{j}^{\mu},
$$

where $i, j$ label the sites in the array and $S_{i}^{\mu}$ the spin components at site $i$.

In the transverse case $\boldsymbol{h}=\left(0,0, h_{z}\right)$, the Hamiltonian commutes with the $S_{z}$ spin parity $P_{z}=e^{i \pi \sum_{i}\left(S_{i}^{z}+1 / 2\right)}=$ $\prod_{i}\left(-2 S_{i}^{z}\right)$, implying that the exact GS will have a definite parity if non-degenerate. In particular, in finite chains of $N$ spins with first neighbor couplings and anisotropy $\chi=J_{y} / J_{x} \in(0,1]$, the exact GS, which can be analytically obtained through the Jordan-Wigner fermionization 61, 80], will exhibit $N / 2$ parity transitions as the field $h_{z}$ increases from 0 , where the lowest levels of each parity cross, the last one at the transverse factorizing field 61] $h_{z s}=\sqrt{J_{y} J_{x}}=J_{x} \sqrt{\chi}$. These transitions are reminiscent of the $N / 2$ magnetization transitions of the $X X$ case $\chi=1$ [53, 81, where $H$ commutes with the $z$ component of the total spin $S^{z}=\sum_{i} S_{i}^{z}$.

At the factorizing field, the two crossing states generate a two dimensional GS subspace which is spanned, remarkably, by completely separable ground states $|\Theta\rangle=$ $|\theta, \ldots, \theta\rangle$ and $|-\Theta\rangle=P_{z}|\Theta\rangle=|-\theta, \ldots,-\theta\rangle$ in the ferromagnetic case $J_{x}>0$, where $|\theta\rangle=e^{-\imath \theta S_{y}}|\downarrow\rangle$ is the single spin state forming an angle $\theta$ with the $-z$ direction and $\cos \theta=h_{s z} / J_{x}=\sqrt{\chi}$. Hence, at this point the system possesses two completely separable parity breaking degenerate ground states. Yet, the exact GS side-limits at this point are provided by the definite parity combinations 61]

$$
\left|\Theta_{ \pm}\right\rangle=\frac{|\Theta\rangle \pm|-\Theta\rangle}{\sqrt{2(1 \pm\langle-\Theta \mid \Theta\rangle)}}
$$

approached for $h_{z} \rightarrow h_{z s}^{ \pm}$, which are entangled states. They lead to common reduced states $\rho_{\theta \pm}$ for any spin pair $i \neq j$ [47, 61], becoming both identical with $\rho_{\theta}=$ $(|\theta \theta\rangle\langle\theta \theta|+|-\theta-\theta\rangle\langle-\theta-\theta|) / 2$ if the overlap $\langle-\Theta \mid \Theta\rangle=$ $\cos ^{N} \theta$ is neglected (it is negligible if $N$ and $\theta$ are not too small). This is a separable mixed state, therefore leading to a zero concurrence (and hence zero entanglement of formation 78]) for any pair, as seen in the bottom left panel of Fig. 2 The concurrence actually approaches small common side limits $C_{ \pm}=\frac{\chi^{n / 2-1}(1-\chi)}{1 \pm \chi^{n / 2}}$ if the overlap is preserved [61, 64, not appreciable in the scale of fig.

\section{2}
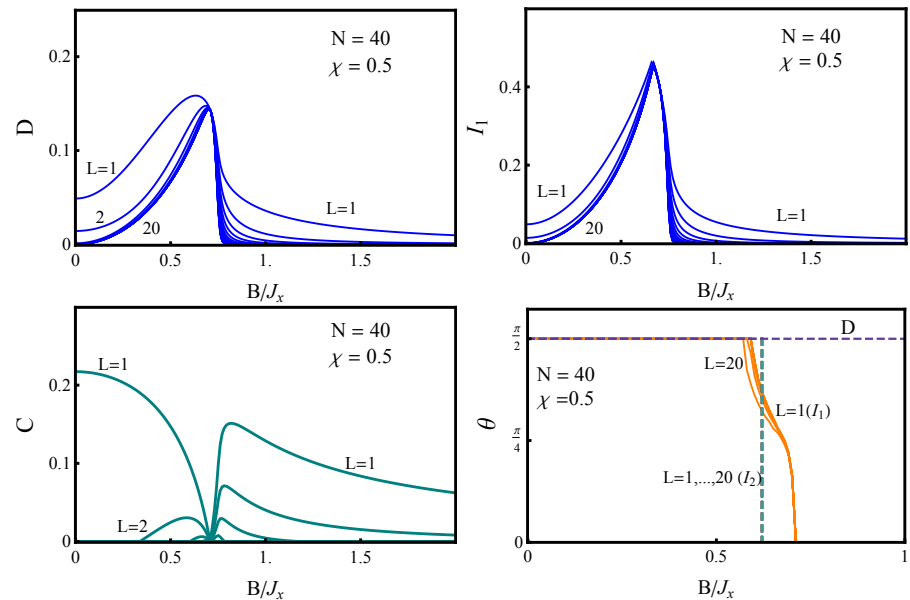

FIG. 2. The quantum discord (top left), the information deficit (top right), the concurrence (bottom left) and the angle $\theta$ determining the minimizing spin measurement of the first two quantities (bottom right) for reduced states of spin pairs in the exact ground state of a spin $1 / 2$ chain with first neighbor anisotropic $X Y$ coupling as a function of the transverse field $\left(B=h_{z}\right)$. $L$ indicates the separation between the spins of the pair ( $L=1$ denotes first neighbors) while $N$ is the number of spins and $\chi=J_{y} / J_{x}$ the anisotropy. The angle $\theta$ is that formed between the measurement direction and the $z$ axis in the $x z$ plane, which is constant for $D(\theta=\pi / 2)$ but experiences an $x \rightarrow z$ transition in the information deficits $I_{1}$ (solid line) and $I_{2}$ (dashed line), which is sharp in the latter. All quantities reach full range at the factorizing field $B=J_{x} \sqrt{\chi}$, with common $L$-independent limits, which are negligible in the case of the concurrence but finite for the discord and information deficit.

However, $\rho_{\theta}$ is a discordant state for $\theta \in(0, \pi / 2)$, leading to appreciable finite limits of the quantum discord $D$ and the information deficit $I_{1}$ at the factorizing field, as seen in the top panels of Fig. 22 These limits can be analytically determined from the previous expression for $\rho_{\theta}$ 47, 76] and are independent of the separation $L$. Moreover, these quantities actually attain their maximum values in the vicinity of this point, remaining appreciable for all $h_{z}<h_{z s}$, since in this sector the reduced state of any pair in the exact GS will be essentially $\rho_{\theta}$ (with a fielddependent $\theta$ ) plus smaller corrections. Let us note that in the cyclic chain considered, the reduced pair states $\rho_{i j}$ depend just on the separation $L=|i-j|$, implying $D(i \mid j)=D(j \mid i)=D$ and $I_{f}^{i}\left(\rho_{i j}\right)=I_{f}^{j}\left(\rho_{i j}\right)=I_{f} \forall i \neq j$.

These results remain strictly valid for arbitrary range couplings with a common anisotropy $\chi=J_{y}^{i j} / J_{x}^{i j}$ in the ferromagnetic case $J_{x}^{i j}>0$ 61, including dimer-type chains 65, since they also exhibit a factorizing field with the same factorized states. They hold as well in the antiferromagnetic case $J_{x}<0$ for first neighbor couplings in a spin chain, since for a transverse field it can be mapped to the ferromagnetic case by a local rotation at even sites, which leads to $|\theta \theta \ldots\rangle \rightarrow|\theta,-\theta, \ldots\rangle$ in the factorized states and in $\rho_{\theta}$. The same reduced state $\rho_{\theta}$ also follows 
from the mixture $\frac{1}{2}\left(\left|\Theta_{+}\right\rangle\left\langle\Theta_{+}|+| \Theta_{-}\right\rangle\left\langle\Theta_{-}\right|\right)$if the overlap is neglected, which is the exact $T \rightarrow 0^{+}$limit at $h_{z s}$ of the thermal state $\propto \exp [-H / k T]$. We remark finally that in the thermodynamic limit $N \rightarrow \infty$, the lowest states for each parity become degenerate for $h_{z}<h_{c}=\frac{J_{x}+J_{y}}{2}$, so GS correlations actually depend on the choice of GS, the present results applying for the definite parity choice.

As seen in Fig. 22 although the quantum discord $D$ and the information deficit $I_{1}$ exhibit a similar qualitative behavior, $I_{1}$ shows a more pronounced maximum in comparison with $D$. This feature reflects the transition in the orientation $\boldsymbol{k}$ of the local spin measurement minimizing $I_{1}$ as the field increases, which is absent in the quantum discord. This effect can be understood from the expressions (28)-29) for the quadratic deficit $I_{2}$, which lead to a sharp $x \rightarrow z$ transition in the optimizing $\boldsymbol{k}$ for all separations as the maximum eigenvalue of $M_{2}$ shifts from that associated with $\boldsymbol{k}=\boldsymbol{e}_{x}$ to that for $\boldsymbol{k}=\boldsymbol{e}_{z}$ as the transverse field increases [29, 76]. In the case of $I_{1}$ such sharp transition is smoothed, as seen in the bottom right panel of Fig. 2 with $\theta$ covering all intermediate values in a narrow field interval centered at the $I_{2}$ measurement transition. In contrast, the quantum discord prefers a spin measurement (we consider here projective spin measurements) along the $x$ axis for all transverse fields, for any separation $L$, following the strongest correlation 54, 55., which is along $x$ for $\left|J_{x}\right|>\left|J_{y}\right|$. This is precisely the same measurement minimizing the quadratic conditional entropy, determined by Eqs. (19)-21), since the largest eigenvalue in (20) of the contracted correlation matrix $C^{T} C$ corresponds to $\boldsymbol{k}$ along the $x$ axis for the present anisotropic $X Y$ coupling $\forall h_{z}$ [54, 55].

The measurement transitions of the information deficit reflect, on the other hand, the qualitative change undergone by the reduced state of the pair (essentially by its dominant eigenstate) as the field increases 76. The same $x \rightarrow z$ transition in $I_{1}$ is found in the $X X$ case, where it reflects the transition in the dominant eigenstate of the reduced state of the pair from a Bell state $\frac{|\uparrow \downarrow\rangle+|\downarrow \uparrow\rangle}{\sqrt{2}}$ to the aligned state $|\downarrow \downarrow\rangle$ as the transverse field increases [53]. The main correlation in $C^{T} C$ stays, however, along the $x$ axis. And in spin 1 systems, while the local optimizing measurements become more complex (they are not standard spin measurements), a similar transition pattern is observed in the measurement minimizing the information deficit 77.

In Fig. 3 we depict illustrative results for a nontransverse field $\boldsymbol{h}=\left(h_{x}, 0, h_{z}\right)$ in the $x z$ plane, for an $X Y$ chain with coupling anisotropy $\chi=0.5$ and small spin number $N=8$. As recently shown 67, such chains also exhibit a non-transverse GS factorizing field in the $x z$ plane, whose magnitude is given by

$$
\left|\boldsymbol{h}_{s}\right|=\frac{h_{z s} \sin \theta}{\sin (\theta-\gamma)}
$$

where $h_{z s}=J_{x} \cos \theta$ is the transverse factorizing field, with $\cos \theta=\sqrt{\chi}$ and $\gamma<\theta$ the angle formed by the field with the $z$ axis. In contrast with the transverse case,
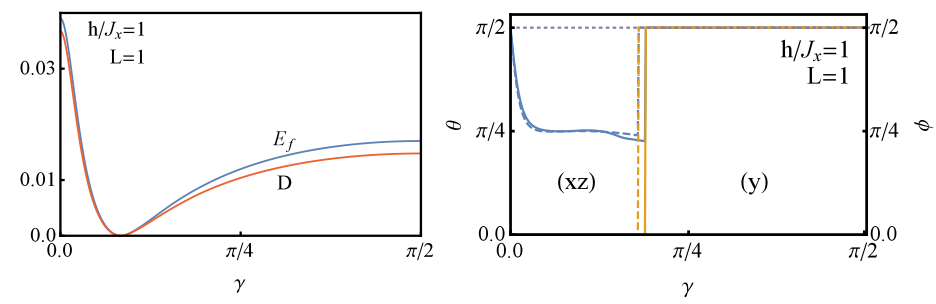

FIG. 3. The quantum discord $D$ and the entanglement of formation $E_{f}$ (left panel), and the angles $\theta, \phi$ determining the minimizing spin measurement direction $\boldsymbol{k}=$ $(\sin \theta \cos \phi, \sin \theta \sin \phi, \cos \theta)$ for $D$ (right panel, solid lines), for a first neighbor pair in a $X Y$ spin chain with $\chi=0.5$, and a non-transverse field $\boldsymbol{h}$ in the $x z$ plane, as a function of the angle $\gamma$ it forms with the $z$ axis, for $|\boldsymbol{h}|=J_{x}$. Here both $D$ and $E_{f}$ vanish at the factorizing field due to the non-degeneracy of the factorized ground state. A transition from the $x z$ plane to the $y$ axis takes place in the minimizing projective measurement as $\gamma$ increases, which can be predicted through the measurement optimizing the quadratic conditional entropy (dashed lines in right panel).

such field is now associated with a non-degenerate separable GS $|\Theta\rangle=|\theta \theta \ldots\rangle$, as parity symmetry no longer holds. It is then seen that both $D$ and the entanglement of formation $E_{f}$ exactly vanish at $\boldsymbol{h}_{s}$, being now smaller than in the previous case since the GS no longer has parity symmetry. Moreover, for first neighbors the reduced pair state is much less mixed than before, and hence $E_{f}$ and $D$ have similar values, with $E_{f}$ slightly larger than $D$, as also occurs for strong transverse fields 47]. For second and more distant neighbors, the behavior of $D$ is qualitatively similar but becomes smaller (and larger than $E_{f}$ ). It should be remarked that $E_{f}$ (and also $D$, $I_{f}$ ) continues to exhibit long range in the vicinity of the non-transverse factorizing field 67].

In addition, the quantum discord now also exhibits a measurement transition if the field is not too small, from the $x z$ plane to the $y$ axis $(\theta=\phi=\pi / 2)$ as the field rotates in the $x z$ plane from the $z$ axis to the $x$ axis. This transition can be understood through the quadratic conditional entropy, as the maximum eigenvalue of the contracted correlation tensor $C^{T} C$ in (20) jumps from the $x z$ block to the $y$ block as the field is rotated, following the main correlation. As verified in the right panel, the measurement minimizing the quadratic conditional entropy lies very close to that minimizing the von Neumann based quantum discord. In contrast, even though the information deficit (not shown) still exhibits a behavior similar to that of $D$, the associated minimizing measurement tends to align with the field for strong $|\boldsymbol{h}|$, deviating again considerably from that minimizing the quantum discord. 


\section{CONCLUSIONS}

We have first described a consistent extension to general concave entropic forms of the measurement dependent von Neumann conditional entropy for bipartite quantum systems. This extension, while providing a general characterization of the average information gain after such measurement, enables the use of simple entropic forms like the quadratic entropy, for which a closed evaluation of the minimizing measurement (leading to maximum purity gain) in terms of the correlation tensor becomes feasible for general states of qudit-qubit systems. Such solution admits a simple geometrical picture and allows to capture the main features of the projective measurement minimizing the quantum discord, which is then seen to follow essentially the direction of maximum correlation. In contrast, that minimizing the information deficit is essentially a least disturbing local measurement, and can then exhibit significant differences with the latter. The entropic generalization of the one way information deficit was also described, and for the quadratic entropy a closed evaluation for qudit-qubit states becomes again feasible, which allows to identify the previous differences.

When considered in spin pairs immersed in finite $X Y$ spin chains, both quantities, discord and information deficit, exhibit similar trends although with significant differences in the behavior of their optimizing measurements, which can be understood and predicted with the closed evaluations for the quadratic case. For transverse fields, these quantities exhibit appreciable values and long range for fields $h<h_{c}$ in the exact definite parity ground state, reaching full range and becoming independent of the pair separation in the vicinity of the factorizing field. A measurement transition takes place in the information deficit, which is absent in the quantum discord. In contrast, for non-transverse factorizing fields parity symmetry is broken and these quantities become smaller, strictly vanishing at factorization. Measurement transitions can occur in both quantities.

\section{ACKNOWLEDGEMENT}

The authors acknowledge support from CONICET (NG,NC,MC) and CIC (RR) of Argentina.
[1] K. Modi et al, Rev. Mod. Phys. 84, 1655 (2012).

[2] G. Adesso, T.R. Bromley, M. Cianciaruso, J. Phys. A 49, 473001 (2016).

[3] B. Schumacher, Phys. Rev. A 512738 (1995).

[4] C.H. Bennett, H.J. Bernstein, S. Popescu, B. Schumacher, Phys. Rev. A 53, 2046 (1996).

[5] M.A. Nielsen and I. Chuang, Quantum Computation and Quantum Information, Cambridge Univ. Press (2000).

[6] S. Haroche and J.M. Raimond,Exploring the Quantum, Oxford Univ. Press, Oxford (2007).

[7] C.H. Bennett et al., Phys. Rev. Lett. 70, 1895 (1993); Phys. Rev. Lett. 76, 722 (1996).

[8] R. Josza and N. Linden, Proc. R. Soc. A459, 2011 (2003).

[9] G. Vidal, Phys. Rev. Lett. 91, 147902 (2003).

[10] R.F. Werner, Phys. Rev. A 40, 4277 (1989).

[11] H. Ollivier and W.H. Zurek, Phys. Rev. Lett. 88, 017901 (2001).

[12] L. Henderson and V. Vedral, J. Phys. A 34, 6899 (2001).

[13] V. Vedral, Phys. Rev. Lett. 90, 050401 (2003).

[14] W.H. Zurek, Phys. Rev. A 67012320 (2003).

[15] E. Knill, R. Laflamme, Phys. Rev. Lett. 81, 5672 (1998).

[16] A. Datta, A. Shaji, and C.M. Caves, Phys. Rev. Lett. 100, 050502 (2008).

[17] A. Datta, S.T. Flammia and C.M. Caves, Phys. Rev. A 72, 042316 (2005).

[18] M. Koashi, and A. Winter, Phys. Rev. A 69, 022309 (2004).

[19] A. Datta and S. Gharibian, Phys. Rev. A 79, 042325 (2009).

[20] A. Shabani and D.A. Lidar, Phys. Rev. Lett. 102, 100402 (2009).

[21] K. Modi et al, Phys. Rev. Lett. 104, 080501 (2010).
[22] A. Ferraro et al, Phys. Rev. A 81, 052318 (2010).

[23] F.F. Fanchini et al, Phys. Rev. A 84, 012313 (2011).

[24] M. Horodecki, et al, Phys. Rev. A 71,062307 (2005).

[25] J. Oppenheim et al, Phys. Rev. Lett. 89180402 (2002).

[26] A. Streltsov, H. Kampermann, and D. Bruß, Phys. Rev. Lett. 106, 160401 (2011).

[27] B. Dakić , V. Vedral, and C. Brukner, Phys. Rev. Lett. 105, 190502 (2010).

[28] R. Rossignoli, N. Canosa, L. Ciliberti, Phys. Rev. A 82, $052342(2010)$.

[29] R. Rossignoli, N. Canosa, L. Ciliberti, Phys. Rev. A 84, 052329 (2011).

[30] D. Girolami, T. Tuffarelli, G. Adesso, Phys. Rev. Lett. 110240402 (2013).

[31] S. Luo, S. Fu, C.H. Oh, Phys. Rev. A 85032117 (2012).

[32] M. Paula, T.R. de Olivera, M.S. Sarandi, Phys. Rev. A 87064101 (2013).

[33] H. Hu, H. Fan, D.L. Zhou, Phys. Rev. A 87032340 (2013).

[34] T. Nakano, M. Piani, G. Adesso, Phys. Rev. A 88012117 (2013).

[35] T. Baumgratz, M. Cramer, and M.B. Plenio, Phys. Rev. Lett. 113, 140401 (2014).

[36] I. Marvian and R. W. Spekkens, Phys. Rev. A 90, 062110 (2014).

[37] V. Madhok, A. Datta, Phys. Rev. A 83, 032323 (2011).

[38] D. Cavalcanti et al, Phys. Rev. A 83, 032324 (2011).

[39] M. Piani et al, Phys. Rev. Lett. 106, 220403 (2011).

[40] D. Girolami, G. Adesso, Phys. Rev. Lett. 108150403 (2012).

[41] T. Tufarelli et al, Phys. Rev. A 86, 052326 (2012).

[42] Y. Wang, New. J. Phys. 16, 033027 (2014).

[43] R. Dillenschneider, Phys. Rev. B 78, 224413 (2008). 
[44] M.S. Sarandy, Phys. Rev. A 80, 022108 (2009).

[45] J. Maziero et al, Phys. Rev. A 82012106 (2010).

[46] T. Werlang and G. Rigolin, Phys. Rev. A 81, 044101 (2010).

[47] L. Ciliberti, R. Rossignoli, and N. Canosa, Phys. Rev. A 82, 042316 (2010).

[48] T. Werlang et al, Phys. Rev. Lett. 105, 095702 (2010).

[49] T. Werlang, G.A.P Ribeiro, G. Rigolin, Phys. Rev. A 83, 062334 (2011).

[50] B.Q. Liu et al, Phys. Rev. A 83, 052112 (2011).

[51] Y.C. Li, H.Q. Lin, Phys. Rev. A 83, 052323 (2011).

[52] N. Canosa, L. Ciliberti, R. Rossignoli, Int. J. Mod. Phys. B 271345033 (2012).

[53] L. Ciliberti, N. Canosa, R. Rossignoli, Phys. Rev. A 88, 012119 (2013).

[54] N. Gigena, R. Rossignoli, Phys. Rev. A 90, 042318 (2014).

[55] N. Gigena, R. Rossignoli, J. Phys. A 47, 015302 (2014).

[56] J. Kurmann, H. Thomas and G. Müller, Physica A 112, 235 (1982).

[57] T. Roscilde et al., Phys. Rev. Lett. 93, 167203 (2004).

[58] T. Roscilde et al., Phys. Rev. Lett. 94, 147208 (2005).

[59] L. Amico et al., Phys. Rev. A 74, 022322 (2006).

[60] F. Baroni et al., J. Phys. A 40, 9845 (2007).

[61] R. Rossignoli, N. Canosa and J. M. Matera, Phys. Rev. A 77, 052322 (2008).

[62] S. M. Giampaolo, G. Adesso and F. Illuminati, Phys. Rev. Lett. 100, 197201 (2008)

[63] S. M. Giampaolo, G. Adesso and F. Illuminati, Phys. Rev. B 79, 224434 (2009).
[64] R. Rossignoli, N. Canosa and J. M. Matera, Phys. Rev. A 80, 062325 (2009).

[65] N. Canosa, R. Rossignoli and J. M. Matera, Phys. Rev. B 81, 054415 (2010).

[66] S. Campbell, J. Richens, N. Lo Gullo, T. Busch, Phys. Rev. A 88062305 (2013).

[67] M. Cerezo, R. Rossignoli, N. Canosa, Phys. Rev. B 92, $224422(2015)$.

[68] H. Wehrl, Rev. Mod. Phys. 50, 221 (1978).

[69] N. Canosa, R. Rossignoli, Phys. Rev. Lett. 88, 170401 (2002).

[70] C. Tsallis, J. Stat. Phys. 52, 479 (1988); C. Tsallis, Introduction to non-extensive statistical mechanics (Springer, New York, 2009).

[71] R. Filip, Phys. Rev. A 65062320 (2002).

[72] H. Nakazato et al, Phys. Rev. A 85042316 (2012).

[73] T. Tanaka, G. Kimura, H. Nakazato, Phys. Rev. A 87 012303 (2013).

[74] V. Vedral, Rev. Mod. Phys. 74, 197 (2002).

[75] R. Bhatia, Matrix Analysis (Springer, New York, USA, 1997).

[76] N. Canosa, L. Ciliberti, R. Rossignoli, Entropy 17, 1634 (2015).

[77] R. Rossignoli, J.M. Matera, N. Canosa, Phys. Rev. A 86, 022104 (2012).

[78] S. Hill and W.K. Wootters, Phys. Rev. Lett. 78, 5022 (1997); W.K. Wootters, Phys. Rev. Lett. 80, 2245 (1998).

[79] P. Rungta and C.M. Caves, Phys. Rev. A 67, 012307 (2003); P. Rungta et al, Phys. Rev. A 64, 042315 (2001).

[80] E. Lieb, T. Schultz, and D. Mattis, Ann. Phys. (NY) 16, 407 (1961).

[81] N. Canosa, R. Rossignoli, Phys. Rev. A 75, 032350 (2007). 